\title{
NUMERICAL ANALYSIS OF THE NATURAL CONVECTION IN HORIZONTAL ANNULI AT LOW AND MODERATE RA
}

\author{
E. L. M. Padilla, \\ R. Campregher, \\ and $A$. Silveira-Neto \\ Universidade Federal de Uberlândia \\ Faculdade de Engenharia Mecânica \\ Campus Santa Mônica \\ Bloco $1 \mathrm{M}$ \\ 38400-902, Uberlândia, MG, Brasil \\ epadilla@mecanica.ufu.br

\begin{abstract}
The natural convection at low and moderate Rayleigh numbers $(\mathrm{Ra})$ in cylindrical horizontal annuli with imposed temperatures in both surfaces is numerically studied. This flow inside concentric cylinders classic configuration has a wide range of practical and technological applications, which justifies its growing studies efforts. In this work, the governing equations are discretized by the volume finite technique over a staggered grid, with second-order accuracy in space and time. The flow pattern is presented by several Rayleigh numbers, with an analysis of the heat transfer coefficient and flow properties. Furthermore, a three-dimensional field is shown at a moderate $R a$ number. The results showed a good agreement with the experimental data.
\end{abstract}

Keywords: Natural convection, flow inside annuli, 3D flow analysis

\section{INTRODUCTION}

The natural convection between concentric cylinders, also known as concentric annuli, has still receiving the attention of many researches around the world. Indeed, several important engineering applications can be modeled by the correct analyses of this arrangement such as electronic cable cooling systems, nuclear reactors, food processing devices, manifolds of solar energy collectors, etc.

A good overview of the early studies on concentric and eccentric annuli can be seen on Kuehn and Goldstein papers. In an experimental and numerical work (1976), they employed air and water inside an enclosure formed by two copper-made concentric horizontal cylinders having a diameter ratio $\left(D_{o} / D_{i}\right)$ of 2.6 for the experimental procedure and finite difference method for the numerical counterpart. The temperatures records in the experimental apparatus were done by an interferometer. The $R a$ values, based on the gap with, ranged from $2.11 \times 10^{4}$ to $9.76 \times 10^{5}$ and the authors found the fluid to behave in a unsteady pattern at $R a>10^{5}$. In the numerical calculus, the grid chosen was a $16 \times 19$ mesh, and the stream function-vorticity approach to the flow was solved by the SOR method as well as the temperature distribution equation. In an experimental paper (1978), the authors studied the temperature distribution, flow patterns, and local and global heat transfer coefficients to a Rayleigh number as wide as possible. The transition and the onset of the turbulent pattern could be detected and observed on the experimental apparatus, since the Rayleigh numbers were ranged from $10^{1}$ to $10^{6}$. Some years later, Macleod and Bishop (1989) performed, in also an experimental work, several measurements of overall heat transfer rates and profiles of the timeaveraged and fluctuations of the temperature field for fully turbulent natural convection. Furthermore, the authors presented an empirical equation which correlates the heat transfer data that takes into account the Rayleigh number, over the ranges $8 \times 10^{6}$ and $2 \times 10^{9}$, the expansion number $(\beta \Delta T)$, over 0.25 and 1.0 , and the diameter ratio $\left(D_{o} / D_{i}\right)$, from 3.37 to 4.85. Their results indicated that the expansion number must be taken into account, regardless of the Rayleigh number, when predicting the heat transfer rates.

Numerical results of the turbulent flow in a twodimensional domain were presented by Farouk and Güçeri (1982). In their work, the natural convection was considered in the annulus between two horizontal concentric cylinders. The stream-vorticity equation was discretized by finite-difference technique and the turbulence was modeled by the $\kappa-\varepsilon$ approach for Rayleigh numbers above $10^{5}$. Furthermore, the outside to inside diameter ratio was 2.6 and the working flow Prandtl number was 0.721 .

In most of the numerical studies on buoyancydriven flow in an annulus, the flow is assumed to be invariant in the axial direction, leading to a twodimensional approach of the problem. However, in practical applications, the viscous shearing effects at the end walls could led to erroneous results. Vafai and Ettefagh (1991) performed a three-dimensional buoyancy-driven flow simulation in a closed horizontal annulus. They furnished a detailed analysis of the three-dimensional flow and temperature field. Moreover, the effect of the impermeable walls on the flow and the three-dimensional dependency of the Nusselt number were analyzed and discussed.

A comparative analysis of the finite-element (FEM) and finite-difference (FDM) methods for solving the buoyancy-driven flow in two and threedimensional annulus was done by Vafai and Desai (1993). By the time of their study, the computer memory saving was a must and the authors recommended the FEM in order to obtain fairly good results employing less computational resources. However, despite more expensive, the FDM was 
more suitable when a more detailed flow and temperature field information was needed. Nowadays, due to the availability of better computer resources, the options are concentrated basically on the latter and the finite-volume method (FVM).

In another paper, Desai e Vafai (1994) performed the numerical simulation of laminar and turbulent natural convection of both two-dimensional (infinitely long annulus) and three-dimensional (annulus with finite length). For turbulence modeling, the authors used the standard $\kappa-\varepsilon$ model in a domain discretised by the Finite Element Method. This work explored quite well the influence of the axial endwalls on the flow and temperature field. The effect of the several Rayleigh numbers, Prandtl numbers and radius ratio were considered on the heat transfer rates. Their results showed the decrease in heat transfer from the inner and outer cylinder with an increase in the radius ratio, for the same Rayleigh number. The level of turbulence, monitored by the turbulent viscosity values, seemed to diminish with the increase of the Prandtl number. Furthermore, the transition to turbulence got delayed for higher Prandtl number fluids. The analysis of the threedimensionality effects showed that the Nusselt number had a drastic decrease at the end walls, being more substantial in the turbulent regime, due to the prominent damping effect of the end walls at high Rayleigh numbers.

The work by Char and Hsu (1998), on turbulence modeling of natural convection in cylindrical horizontal annuli, proposed a comparison of the different turbulence models, suggesting a better modeling practice. The Rayleigh numbers were varied from $8.02 \times \cdot 10^{5}$ to $1.18 \times \cdot 10^{9}$ and the outer and inner diameter ratio from 2.6 to 4.85 . According to the authors, the nonlinear model gives better results, especially at higher $R a$ numbers.

In the present work, the natural laminar and transitional convection of the flow inside two concentrical cylinders is performed. Furthermore, a detailed analysis of the three-dimensional thermal field and the flow pattern is presented.

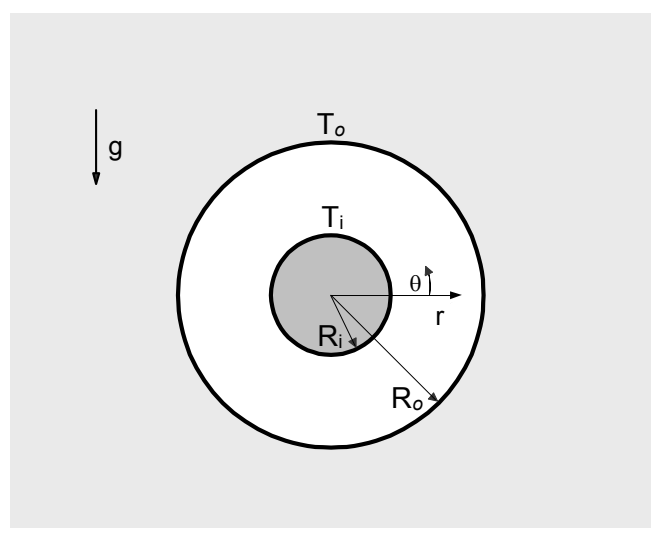

Figure 1. The two concentrical cylinders configuration.

\section{NUMERICAL SOLUTION}

The Navier-Stokes equations were discretized by the Finite-Volume Method (FVM), as proposed by Patankar (1980), and the convective terms were approximated by the Central-Difference Scheme (CDS), see Ferzinger and Perić (1999). The pressurevelocity coupling was done by the second order - Adams-Bashforth - explicit fractional timestep method. So, the new velocity field is guessed by:

$$
\begin{gathered}
\frac{\partial\left(\rho u_{i}\right)}{\partial t}=\frac{3}{2}[A]^{n}-\frac{1}{2}[A]^{n-1}-\frac{\partial p^{n}}{\partial x_{i}}-\rho g_{i} \\
A=-\frac{\partial}{\partial x_{j}}\left(\rho u_{j} u_{i}\right)+\frac{\partial}{\partial x_{j}}\left(\mu \frac{\partial u_{i}}{\partial x_{j}}+\mu \frac{\partial u_{j}}{\partial x_{i}}\right)
\end{gathered}
$$

and corrected by:

$$
u_{i}^{n+1}=u_{i}^{*}-\left(\frac{d t}{\rho}\right)\left(\frac{\partial p^{\prime}}{\partial x_{i}}\right)
$$

where the pressure correction is obtained from:

$$
\frac{\partial}{\partial x_{i}}\left(\frac{\partial p^{\prime}}{\partial x_{i}}\right)^{n+1}=\frac{\rho}{d t}\left[\frac{\partial\left(u_{i}\right)}{\partial x_{i}}\right]
$$

which is the Poisson equation for pressure, solved by the Strongly Implicit Procedure (SIP) solver, as proposed by Stone (1968).

The temperature field is obtained by solving the energy conservation equation, given by:

$$
\frac{\partial(\rho T)}{\partial t}+\frac{\partial\left(u_{j} T\right)}{\partial x_{j}}=\frac{\partial}{\partial x_{j}}\left(\alpha \frac{\partial T}{\partial x_{j}}\right)
$$

Furthermore, the velocity components are located at the volume faces, in a staggered arrangement, which the pressure and the temperature values are located at the cell center.

It is very useful to define the non-dimensional parameter known as Rayleigh number $(R a)$ used in the simulations:

$$
R a=\frac{g \beta\left(T_{i}-T_{o}\right) L^{3}}{v^{2}} P r
$$

where: $\operatorname{Pr}=v / \alpha$ is the Prandtl number, $g$ is the gravity acceleration value, $\beta$ is the thermal expansion coefficient, $v$ is the dynamic viscosity and $\alpha$ the thermal diffusivity. The length $L$ is the difference between the outer and the inner cylinders radii, $L=R_{o}-R_{i}$, as can be seen on the Fig. 1 . In the same sense, the cylinders temperatures are defined by $T_{o}$ and $T_{i}$. In every simulations the flow was considered incompressible and the buoyancy effects were 
approximated by the Boussinesq hypothesis, i.e., the effect is only due to the $T_{o}$ and $T_{i}$ difference, the remaining physical properties were kept unchanged.

\section{RESULTS}

In this work, the Rayleigh number was ranged from $10^{2}$ to $10^{5}$. Furthermore, two different geometric configurations were tested: radii ratio $(\eta)$ of 2.0 and 2.6. The aspect ratio $(\Gamma)$ was constant and equal of 1.0. The domain was discretized in a cylindrical coordinate system having $20 \times 80 \times 2$ volumes along the radial, tangential and axial axes, respectively. Also, the grid was disposal in a non-uniform pattern, i.e., finer closer to the inner and outer walls, although distributed uniformly along the radial direction.

The Figure 2 shows the isotherm (column a), velocity fields (column $\mathrm{b}$ ), and the streamlines for $R a$ numbers of $10^{2}, 10^{3}$ and $10^{4}$ for $\eta=2.6$. One can see that for $R a=10^{2}$ the heat transfer process are mainly due to the conduction effect, as the isothermal lines are in a concentric pattern. At $R a=10^{3}$, the advective process becomes stronger, the isothermal loses its circular shape, being stretched upward. At the moderate $R a=10^{4}$, the convective process is the predominate one, inducing the thermal plume formation in the annulus upper part. The flow driven by the temperature difference at $R a=10^{2}$, shows two recirculating symmetric opposite cells centered at $0^{\circ}$ and $180^{\circ}$. At higher $R a$, the recirculating cells centers moves upward as the convective effects become stronger. According to the velocities vector field, one can see the higher velocity magnitude occurs closer to the cylinders walls, moving upward as the Rayleigh number increases. Furthermore, is possible

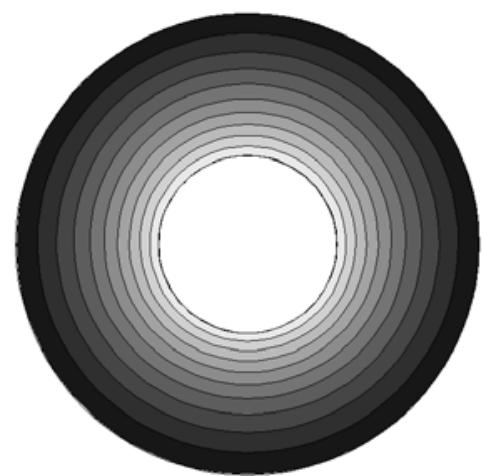

$\mathrm{Ra}=1 \mathrm{e} 02$

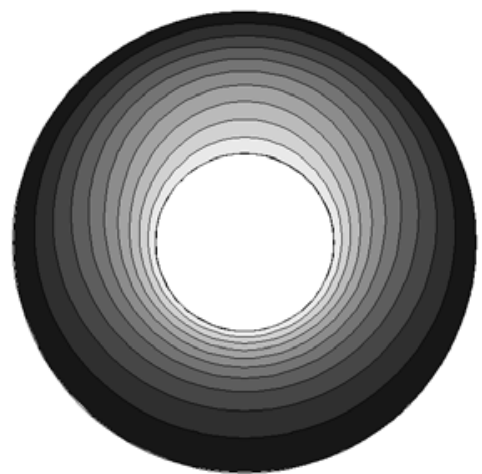

$\mathrm{Ra}=1 \mathrm{e} 03$

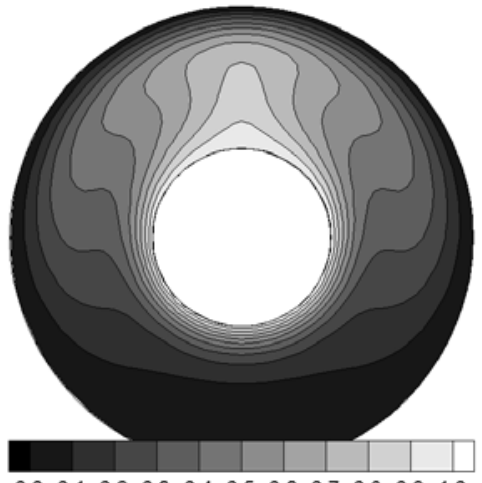

(a)

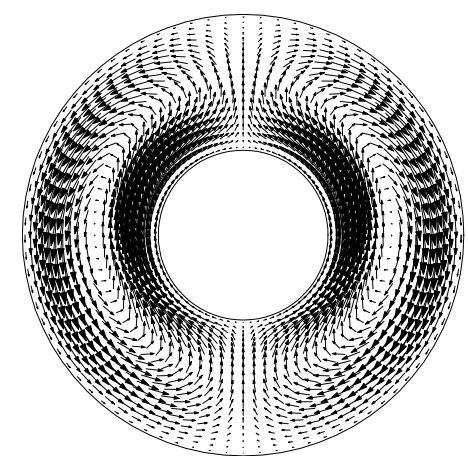

$\mathrm{Ra}=1 \mathrm{e} 02$

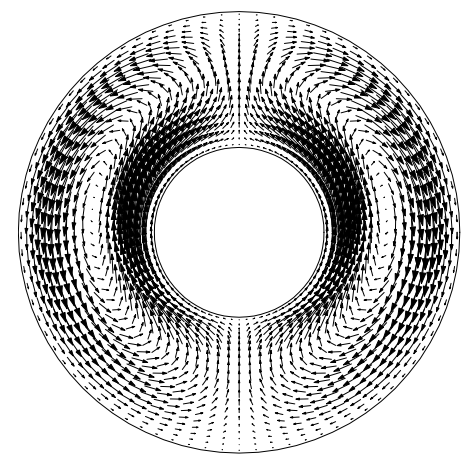

$\mathrm{Ra}=1 \mathrm{e} 03$

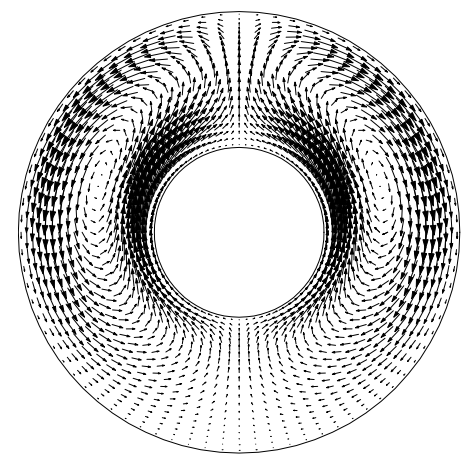

(b)

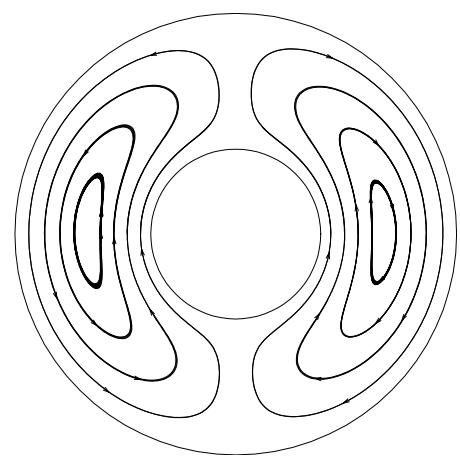

$\mathrm{Ra}=1 \mathrm{e} 02$

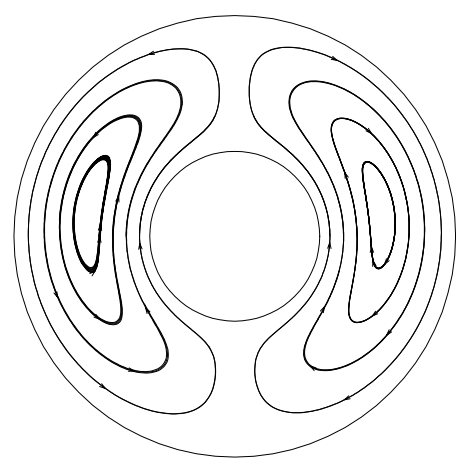

$\mathrm{Ra}=1 \mathrm{e} 03$

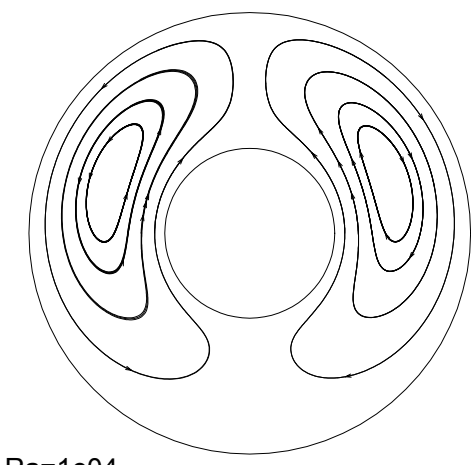

(c)

Figure 2. Isotherms (a), vector field (b) and streamlines (c) at three values of $\operatorname{Ra}, \operatorname{Pr}=0.71, \eta=2.6, \Gamma=1.0$. 

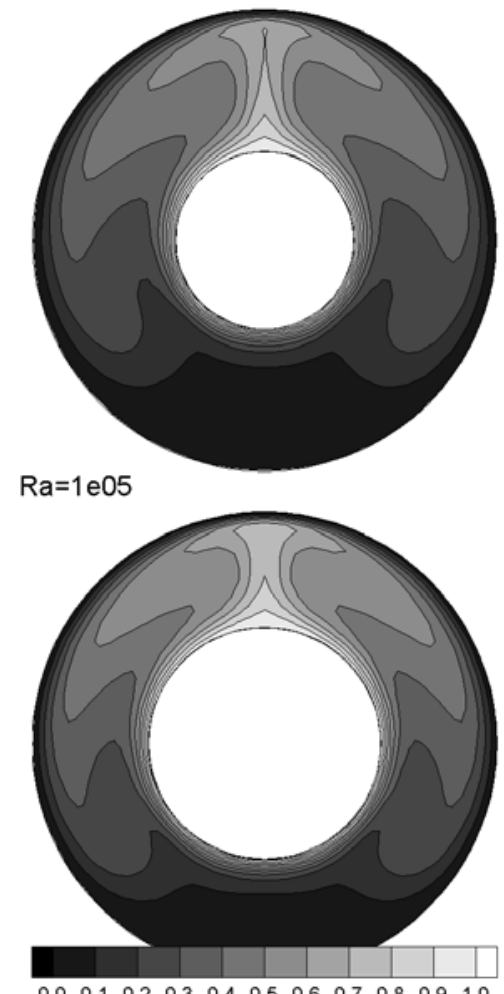

$\begin{array}{llllllllllll}0.0 & 0.1 & 0.2 & 0.3 & 0.4 & 0.5 & 0.6 & 0.7 & 0.8 & 0.9 & 1.0\end{array}$

(a)
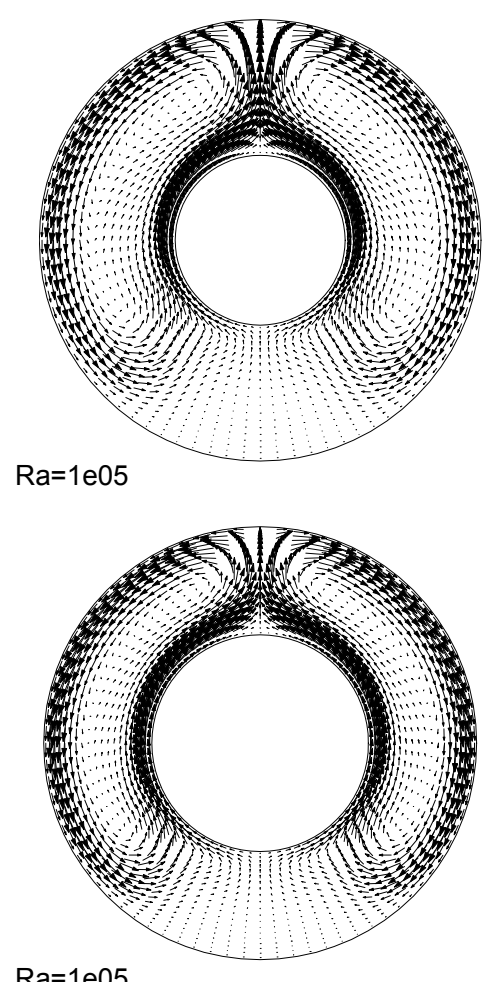

(b)
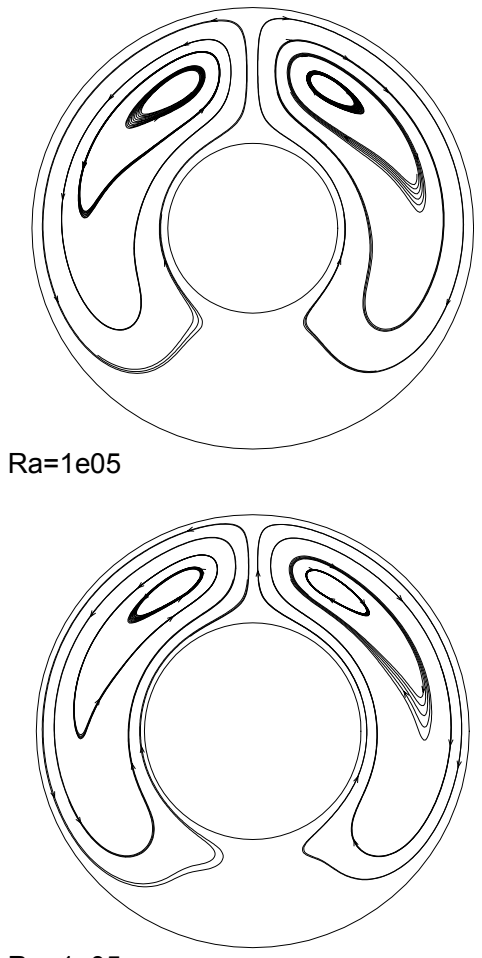

(c)

Figure 3. Isotherms (a), vector field (b) and streamlines (c) at three values of $R a$ and two values of $\eta$ (2.6 and 2.0), $\operatorname{Pr}=0.71, \Gamma=1.0$.

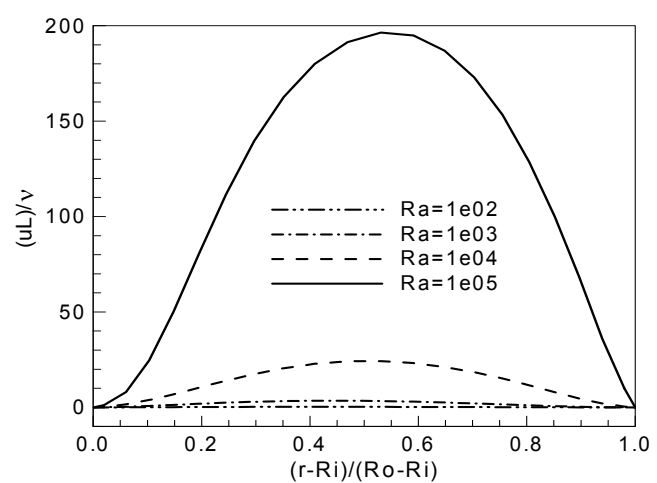

(a)

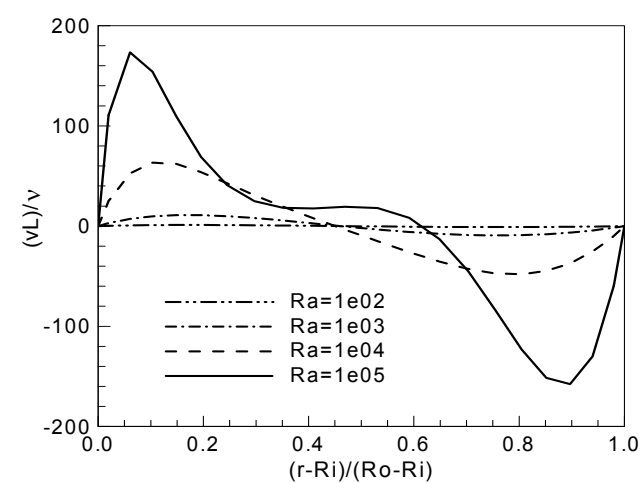

(b)

Figure 4. Velocity distribution at four values of $\operatorname{Ra}, \operatorname{Pr}=0.71, \eta=2.6, \Gamma=1.0$, radial velocity at $\theta=90^{\circ}$ (a), angular velocity at $\theta=0^{\circ}(\mathrm{b})$.

to see that for $90^{\circ}$ and $270^{\circ}$ there is only the radial velocity component and the onset of a stagnation region at the annulus lower part.

In the Figure 3, the same properties before mentioned, but at $R a=10^{5}$ for $\eta=2.0$ (lower row) and 2.6 (upper row). In this configuration, the thermal plume becomes narrower as the thermal boundary layer becomes thicker at the inner cylinder wall. The recirculating flow core, once at the horizontal center line, has moved far upward, tending to the $90^{\circ}$ position. Also, the velocity vectors are concentrated closer to the cylinders walls and, as a consequence, the boundary layer becomes thicker. The plume for $\eta=2.6$ geometric configuration, is narrower than for $\eta=2.0$ due to the greater gap, as can be seen on the left column. On the right column, one can see at the annulus lower part, the inflexional velocity profile has been forced to the $270^{\circ}$ plane direction, because the recirculation zone is stretched along to the tangential direction.

In the Figure 4 are displayed the radial (Fig. 4a) and the tangential (Fig. 4b) component velocity profiles for four different Rayleigh numbers, from $10^{2}$ to $10^{5}$ in the $\eta=2.6$ geometric configuration. The 
velocity profile at $90^{\circ}$ plane is depicted in the Fig. 4a, where is possible to compare the smaller velocities magnitude among the $R a=10^{2}$ and $10^{3}$ than to those at higher Rayleigh numbers. One can see, also, that at $R a=10^{5}$, the inflexional velocity profile occurs above the half way between the inner and the outer cylinder gap. The Fig. $4 \mathrm{~b}$ shows the tangential velocity at $0^{\circ}$ plane. In the same sense, is possible to see the difference in the velocity magnitude and its signal inversion displacement. Although for moderate Rayleigh numbers, the inversion point occurs at the gap half way, one can see that, at $R a=10^{5}$, the inversion locus has been moved towards the outer cylinder. It worth noting that, at $R a=10^{4}$, the velocity gradients near the inner cylinder are steeper than those closer to the outer cylinder, which leads to a non-symmetric velocity profile.
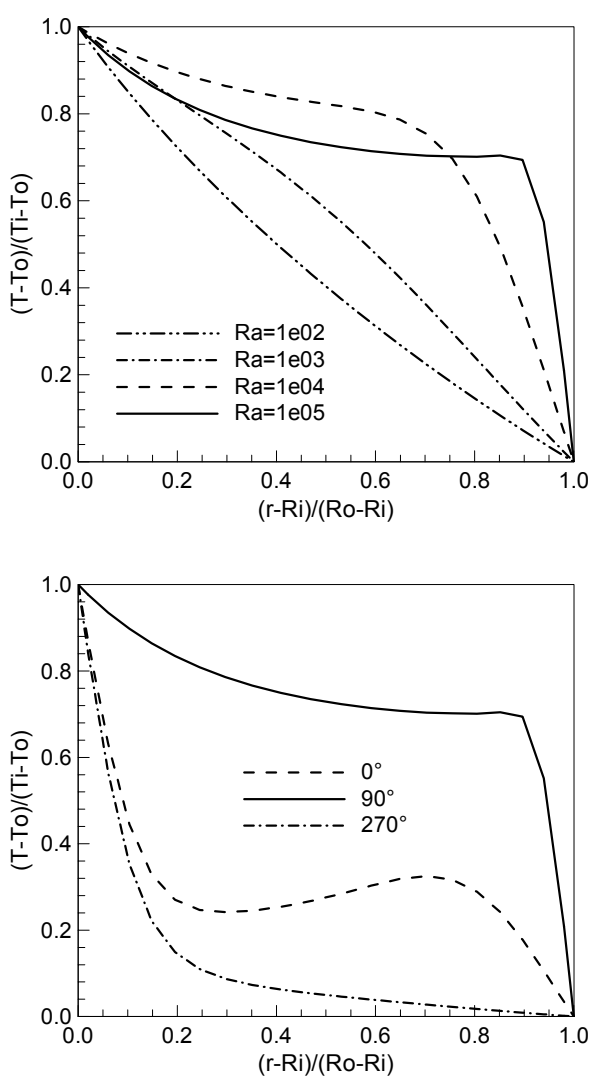

Figure 5. Temperature distribution, $\operatorname{Pr}=0.71, \eta=2.6$, $\Gamma=1.0$, at four values of $R a$ and $\theta=90^{\circ}$ (up), at $R a=10^{5}$ and three values of the $\theta$ (down).

The Figure 5 depicts the temperature profile along the annulus radial axis for $\eta=2.6$ geometric configuration. In the Fig. 5(up), the cutting plane studied is at $90^{\circ}$ and the profiles represent the four Rayleigh numbers, from $10^{2}$ to $10^{5}$. As mentioned before, the temperature distribution at $R a=10^{2}$ indicates a quasi-linear pattern along the gap, which indicates that heat transfer process is mainly due to the conduction phenomena, as a consequence, the temperature gradients are approximately constant. At
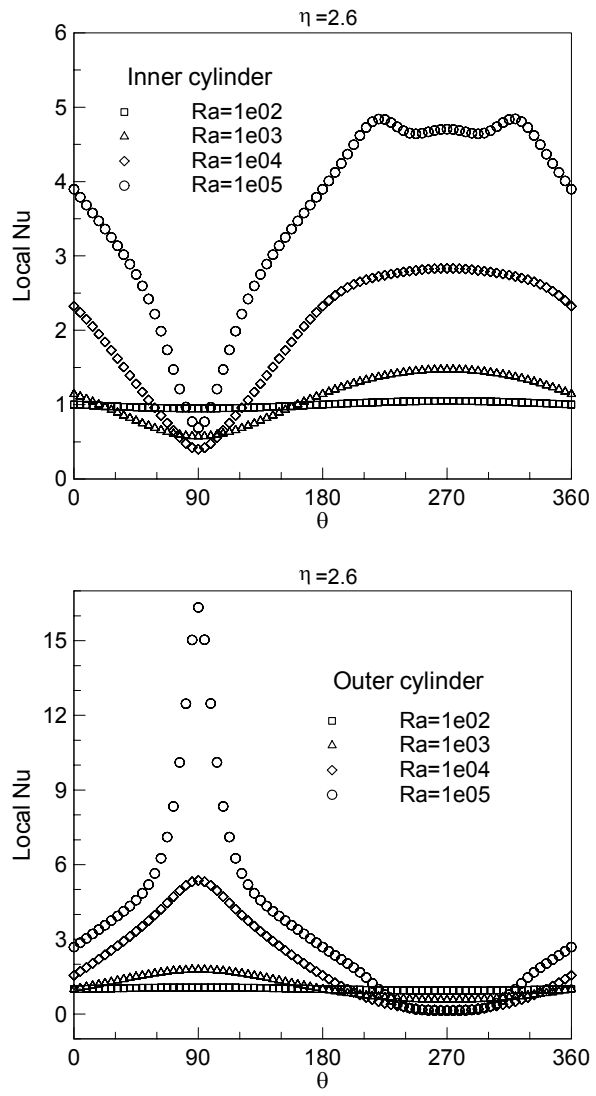

Figure 6. Local Nusselt number distribution at four values of $R a, P r=0.71, \Gamma=1.0$, inner cylinder (up), outer cylinder (down).

Rayleigh numbers of $10^{4}$ and $10^{5}$, one can note the very high temperature gradients closer to the outer cylinder wall. The Fig. 5(down) depicts temperature profiles at $0^{\circ}, 90^{\circ}$, and $270^{\circ}$, for $R a=10^{5}$. It is important to note the temperature transport effect, performed by the thermal plume at $90^{\circ}$. The hot flow was kept warmer at closer distance to the outer cylinder wall. So, the temperature gradients were steeper in this region. In the opposite direction $\left(270^{\circ}\right)$, the temperature profile is smoother, due to the almost stagnated flow. On the hand, at the $0^{\circ}$ plane, one can see the temperature inflexional pattern, mainly due to the recirculating flow driven by the greater plume momentum. As the heat increases, a larger amount of hot flow is transported, as can be seen on the Fig. $3 \mathrm{a}$.

It is very important to define the local $N u$ number over the inner and outer cylinder, respectively, by the equations below:

$$
N u_{i}=\left.R_{i} \ln \left(\frac{R_{o}}{R_{i}}\right) \frac{\partial T}{\partial R}\right|_{r=R_{i}}, N u_{o}=\left.R_{o} \ln \left(\frac{R_{o}}{R_{i}}\right) \frac{\partial T}{\partial R}\right|_{r=R_{o}}
$$


Furthermore, the average Nusselt number $(\overline{N u})$ is evaluated by an arithmetic mean of the Eq. (6).

In the Figure 6, the local Nusselt number, in the inner (up) and outer cylinder (down), is depicted for geometrical configuration of $\eta=2.6$. As can be seen, the Nusselt number distribution, along the inner cylinders surface, attains its lower value at $90^{\circ}$. It is important to mention that, for $R a=10^{2}$, the Nusselt drop behavior at $90^{\circ}$ also occurs, but is masqueraded due to the higher Rayleigh numbers graph scale. At $R a=10^{5}$, one can see the slight $N u$ drop down pattern between $220^{\circ}$ and $320^{\circ}$, which coincides with the larger quasi-static zone at the annuli lower part. The differences between the maximum and minimum local $N u$ number, and the $N u$ curve shape, along the inner cylinder diameter are similar to found on $\eta=2.0$ geometric configuration. At the outer cylinder, the local $N u$ number shows an opposite pattern, i.e., attains its maximum at $90^{\circ}$ and minimum at $180^{\circ}$ for every curve, although for moderate $R a$

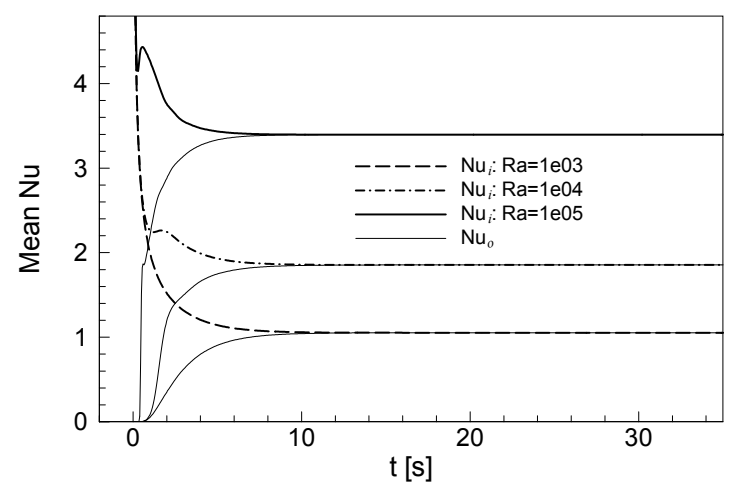

Figure 7. Transient results for average inner and outer $N u$ at several $R a$ and $\eta=2.0$.

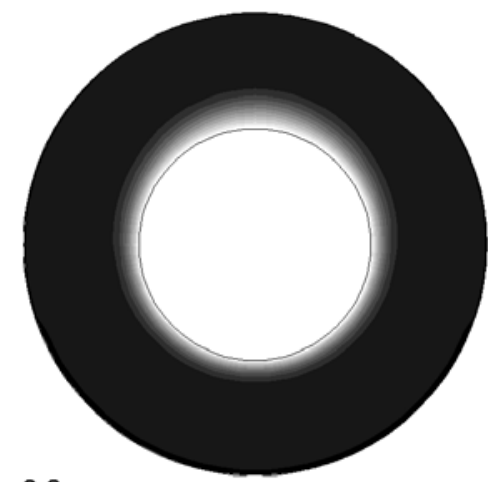

$0.2 \mathrm{~s}$
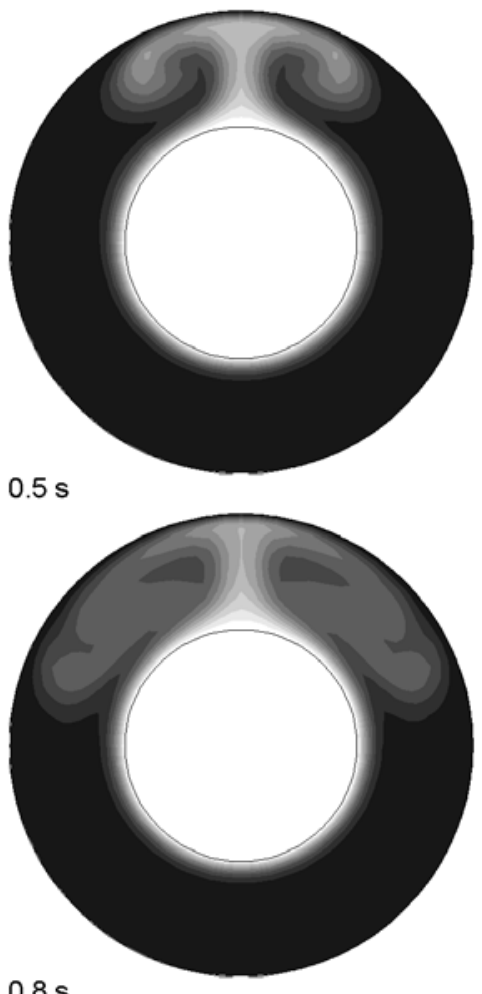

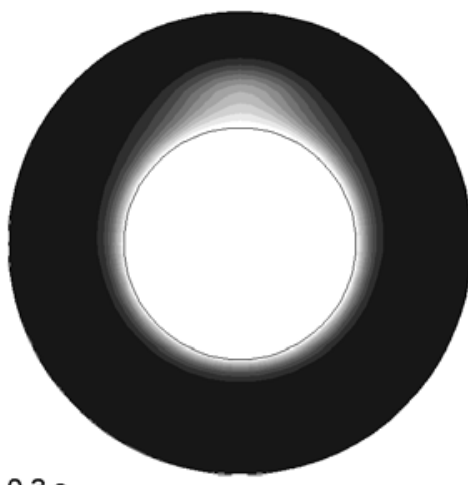

$0.3 \mathrm{~s}$

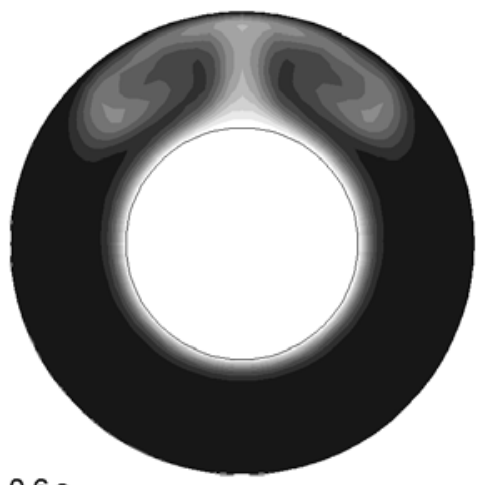

$0.6 \mathrm{~s}$

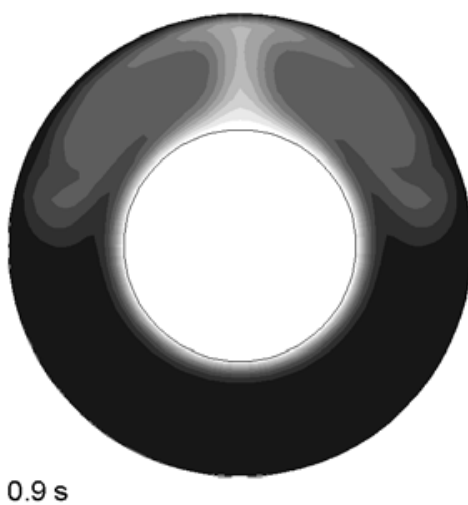

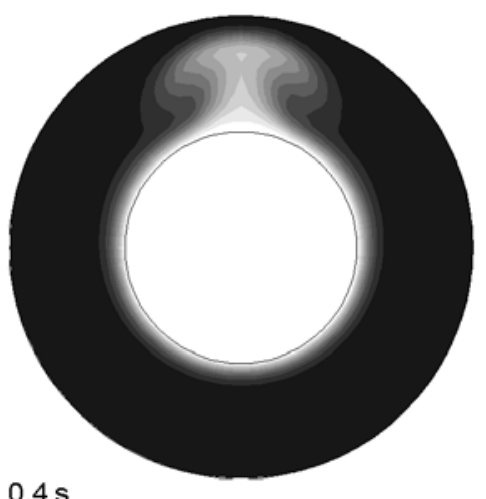

$0.4 \mathrm{~s}$
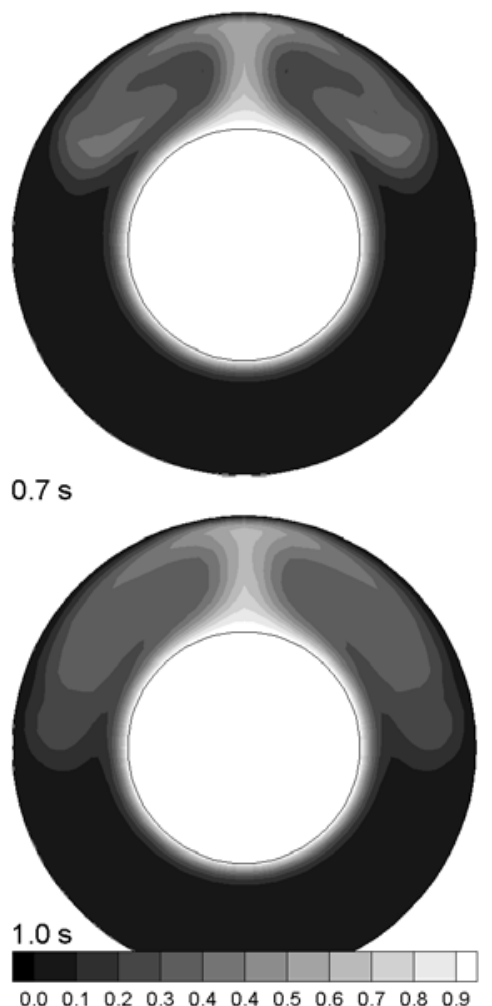

Figure 8. Isotherms (a), vector field (b) and streamlines (c) at three values of Ra and two values of $\eta$ (2.6 and 2.0), $\operatorname{Pr}=0.71, \Gamma=1.0$. 
numbers, the $N u$ number, and as a consequence, the heat transfer is almost null.

The Figure 7 shows the average Nusselt number along the simulation time. It is very interesting to note that the energy balance between inner and outer cylinders tend to converge to the same value, showing the good accuracy of the temperature field solution. As the Rayleigh number increases, the time required to achieve the inner and outer cylinders average $N u$ convergence becomes smaller. This can be explained by the advective process predominance over the diffusive one. One observe that at $R a=10^{4}$ and with more intensity at $R a=10^{5}$ a peak on the inner cylinder $N u$ value. This behavior can be explained by the Fig. 8, that shows the flow time development at $R a=10^{5}$ in the period related to the plume formation. At higher $R a$ numbers, the plume acquires more momentum which leads the flow to suddenly rise. Due to the mass conservation, a portion of cold flow takes the hot flow space that has risen with the plume. As the $R a$ increases, more cold flow takes into contact to the hotter inner cylinder, consequently, more prominent the peak will be.

In order to compare the numerical code results for local properties, that is, local temperatures and Nusselt number, against experimental data (Kuehn and Goldstein, 1976) found in the literature, a test was run at $R a=4.7 \times 10^{4}$ for $\eta=2.6$, and can be seen in the Fig. 9. In the Fig. 9(up), one can see the nondimensional temperature profiles along the annulus radii for $\theta=0^{\circ}, 90^{\circ}$ and $270^{\circ}$. The local $N u$ number for the inner and outer cylinders is depicted in the Fig. 9(down). It is possible to see in both figures a very good agreement between the numerical and the experimental values.

The average Nusselt number $(\overline{N u})$ vs. Rayleigh number, and compared against the experimental and numerical data by Kuehn and Goldstein (1976), is plotted in the Fig. 10. Although the present work both geometrical configurations are depicted, only the $\eta=2.6$ configuration is compared against the latter authors' results. In the Kuehn and Goldstein's work, the experimental results were ranged from $R a=2.11 \times 10^{4}$ to $9.56 \times 10^{4}$ and the numerical were ranged from $R a=10^{2}$ to $7.0 \times 10^{4}$. In this paper, several tests were run at the same $R a$ numbers from those authors in order to get a more accurate comparative data set. There is a very good agreement between the experimental and numerical results and this work's results for every $R a$ numbers considered. It is possible to see, also, that the heat transfer coefficient for $\eta=2.0$ is slightly lower than for $\eta=2.6$. Accordingly to the literature (Powe et al, 1969), the flow becomes unstable at $R a$ numbers close to $10^{5}$, that was confirmed in these additional tests.

In the Figure 11, one can see the onset of axial velocity values at $R a=10^{5}$ for $\eta=2.0$ and $\eta=2.6$. It is important to note that laminar flow is essentially two-dimensional so, the onset of such axial velocity components does not affect the flow stability due to its very small magnitude.
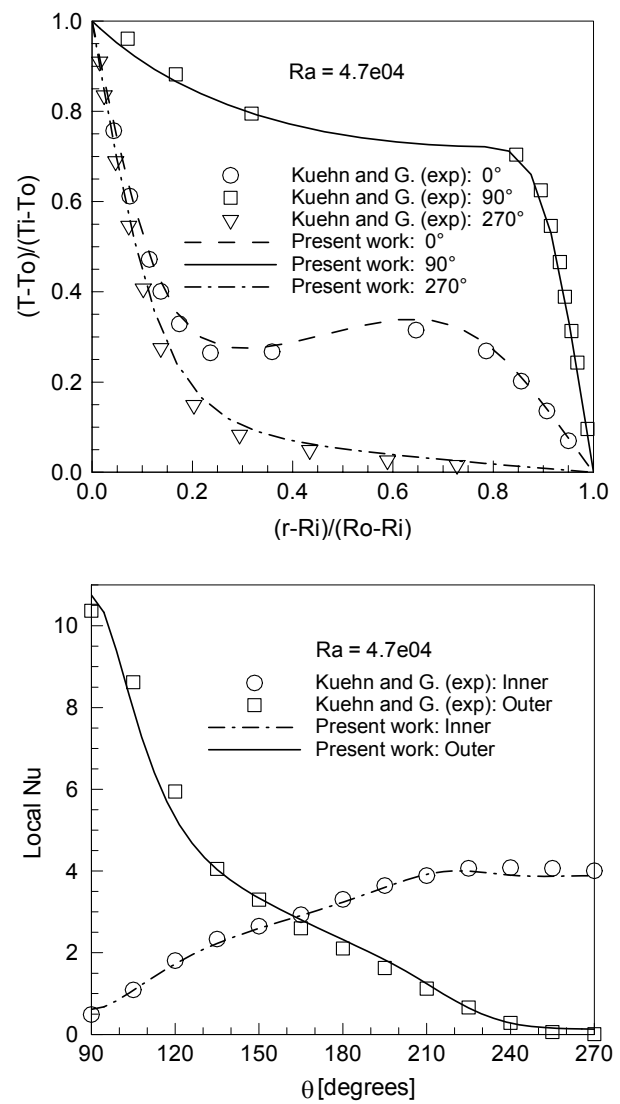

Figure 9. Comparison with experimental dates at $R a=4.7 \times 10^{4}, P r=0.71, \eta=2.6, \Gamma=1.0$, temperature distribution (up), local Nusselt number (down).

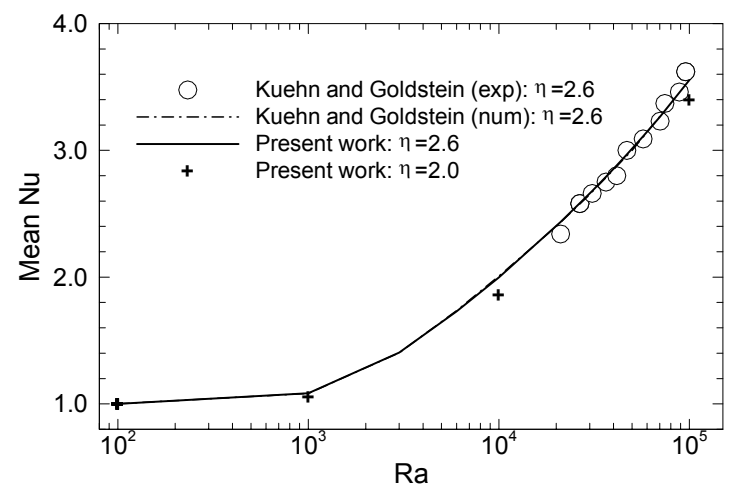

Figure 10. Comparison of mean Nusselt number with experimental and theoretical dates of Kuehn and Goldstein.

\section{CONCLUSIONS}

It is important to note that the results presented in this work show a very good agreement with the experimental and numerical data as well as the flow field pattern found in the literature for laminar 
regimes. Moreover, the energy balance between the inner and outer cylinders, along the simulation time, was very good, as can be seen in the Fig. 7. The flow three-dimensional formulation employed allowed to quantify the axial velocity components, that come up around $R a=10^{5}$. The numerical code presented here with the sub-grid scale turbulence modeling added will be used in order to investigate the transition phenomena in the near future.
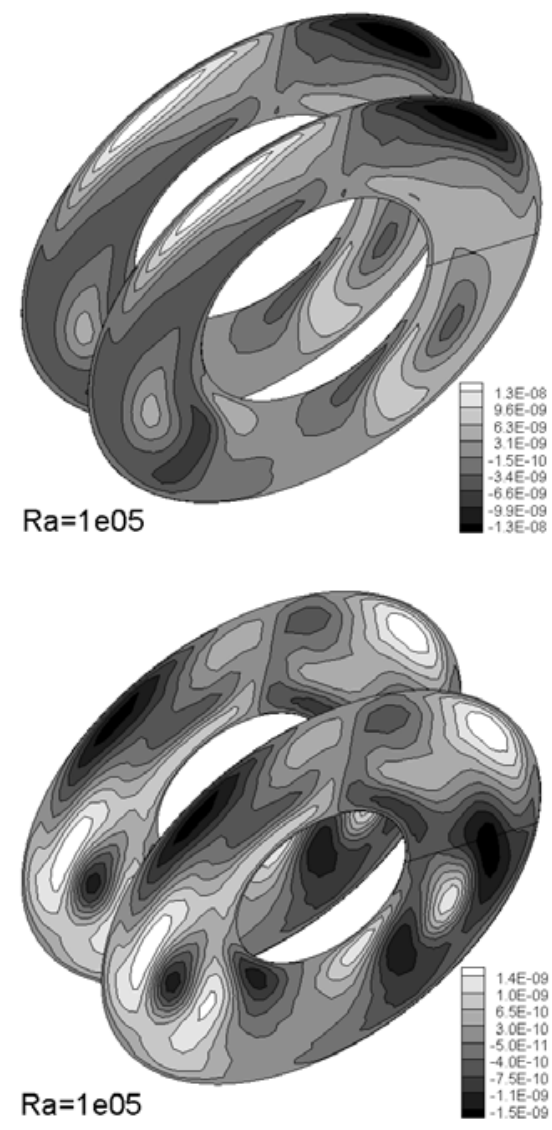

Figure 11. Isovalues of the axial velocity at Rayleigh number of $10^{5}, \operatorname{Pr}=0.7, \Gamma=1.0, \eta=2.0$ (up),

$$
\eta=2.6 \text { (down). }
$$

\section{ACKNOWLEDGEMENTS}

The authors wish to thank the $\mathrm{CNPq}$ and CAPES for the financial support.

\section{REFERENCES}

Char, M., and Hsu, Y. H., 1998, Comparative Analysis of Linear and Nonlinear Low-ReynoldsNumber Eddy Viscosity Models to Turbulent Natural Convection in Horizontal Cylindrical Annuli, Numerical Heat Transfer, vol. 33, pp 191-206.

Desai, C. P., and Vafai, K., 1994, An Investigation and Comparative Analysis of Two and Three-Dimensional Turbulent Natural Convection in a Horizontal Annulus, International Journal of Heat and Mass Transfer, vol. 37, pp 2475-2504.
Farouk, B., and Güçeri, S. I., 1982, Laminar and Turbulent Natural Convection in the Annulus Between Horizontal Concentric Cylinders, Journal of Heat Transfer, vol. 104, pp 631-636.

Ferzinger, J. H., and Peric, M., 1999, Computational methods for fluid dynamics, $2^{\text {nd }}$ Ed., Springer.

Kuehn, T. H., and Goldstein, R. J., 1976, An Experimental and Theoretical Study of Natural Convection in the Annulus Between Horizontal Concentric Cylinders, Journal of Fluid Mechanics, vol. 4, pp 695-719.

Kuehn, T. H., and Goldstein, R. J., 1978, An Experimental Study of Natural Convection Heat Transfer Concentric and Eccentric Horizontal Cylindrical Annuli, Journal of Heat Transfer, vol. 100, pp 635-640.

Macleod, A. E., and Bishop, E. H., 1989, Turbulent Natural Convection of Gases in Horizontal Cylindrical Annuli at Cryogenic Temperatures, International Journal of Heat and Mass Transfer, vol. 32, pp 1967-1978.

Patankar, S.V., (1980), "Numerical heat transfer and fluid flow", Hemisphere.

Powe, R. E., Carley, C. T. and Bishop, E. H., 1969, Free Convection Flow Pattern in Cylindrical Annuli, Journal of Heat Transfer, ASME, Aug., pp 310-314.

Stone, H. L., 1968, Iterative Solutions of Implicit Approximations of Multidimensional Partial Differential Equations, SIAMJ Num. Anal., vol. 5, pp 530-558.

Vafai, K., and Ettefagh, J., 1991, An Investigation of Transient Three-dimensional Buoyancy-Driven Flow and Heat Transfer in a Closed Horizontal Annulus, International Journal of Heat and Mass Transfer, vol. 34, pp 2555-2570.

Vafai, K., and Desai, C. P. 1993, Comparative Analysis of the Finite-Element and Finite-Difference Methods for Simulation of Buoyancy-Induced Flow and Heat Transfer in Closed and Open Ended Annular Cavities, Numerical Heat Transfer, vol. 23, pp 35-59.

\section{Received: May 18, 2006}

Revised: June 18, 2006 Accepted: July 18, 2006 